\title{
Effect of an Oxide Cap Layer and Fluorine Implantation on the Metal-Induced Lateral Crystallization of Amorphous Silicon
}

\author{
Kai Sun, ${ }^{\text {a,z }}$ M. M. A. Hakim, ${ }^{\text {b }}$ R. Gunn, ${ }^{c}$ and P. Ashburn ${ }^{\text {a }}$ \\ ${ }^{a}$ School of Electronics \& Computer Science, University of Southampton, Southampton SO17 1BJ, United Kingdom \\ ${ }^{b}$ Electrical \& Electronic Engineering, East West University, Dhaka 1212, Bangladesh \\ ${ }^{c}$ Oxford Instruments Plasma Technology, Yatton, Bristol BS49 4AP, United Kingdom
}

\begin{abstract}
In this work, we investigate the effect of oxide cap layer on the metal-induced lateral crystallization (MILC) of amorphous silicon The MILC is characterized at temperatures in the range 550 to $428^{\circ} \mathrm{C}$ using Nomarski optical microscopy and Raman spectroscopy. It is shown that better lateral crystallization is obtained when the oxide cap layer is omitted, with the crystallization length increasing by $33 \%$ for a 15 hour anneal at $550^{\circ} \mathrm{C}$. A smaller increase of about $10 \%$ is seen at lower temperatures between $525^{\circ} \mathrm{C}$ and $475^{\circ} \mathrm{C}$ and no increase is seen below $450^{\circ} \mathrm{C}$. It is also shown that the detrimental effect of the oxide cap layer can be dramatically reduced by giving samples a fluorine implant prior to the MILC anneal. Raman spectroscopy shows that random grain growth is significantly less for unimplanted samples without an oxide cap and also for fluorine implanted samples both with and without an oxide cap. The crystallization length improvement for samples without an oxide cap layer is explained by the elimination of random grain crystallization at the interface between the amorphous silicon and the oxide cap layer.
\end{abstract}

(C) 2012 The Electrochemical Society. [DOI: 10.1149/2.038301jss] All rights reserved.

Manuscript submitted October 3, 2012; revised manuscript received November 26, 2012. Published December 7, 2012.

Metal-induced lateral crystallization (MILC) has been widely researched as an alternative to solid-phase crystallization (SPC) of amorphous silicon because of its lower process temperature and higher quality polysilicon (poly-Si) film with higher carrier mobility, larger grain size, and lower defect density. ${ }^{1}$ For MILC, nickel reacts with amorphous silicon $(\alpha-\mathrm{Si})$ to form nickel disilicide and the lateral transport of $\mathrm{Ni}$ induces crystallization of the adjacent amorphous silicon, ${ }^{2,3}$ creating a crystallized region with low Ni contamination suitable for high performance transistors. ${ }^{4}$ In most published work, the $\mathrm{Ni}$ is defined either by a lift-off process $^{5-9}$ or by $\mathrm{Ni}$ deposition in a window opened in a cap layer, which is normally deposited silicon dioxide. ${ }^{10-15}$ So far, no work has been reported that compares the effect of these two different $\mathrm{Ni}$ definition techniques on the lateral crystallization.

In this paper, we study the effect of an oxide cap layer on the nickel-induced lateral crystallization of amorphous silicon, and show that an amorphous silicon film without oxide capping gives better lateral crystallization at temperatures between $475^{\circ} \mathrm{C}$ to $550^{\circ} \mathrm{C}$. It is also shown that the detrimental effects of an oxide cap layer can be eliminated using a fluorine implant.

\section{Experimental Procedure}

The sample structures are shown schematically in Figs. 1a and 1b for Ni patterning by lift-off and oxide window definition, respectively. A $500 \mathrm{~nm}$ thermal silicon dioxide layer was grown on $<100>$ n-type $\mathrm{Si}$ wafers, and a $110 \mathrm{~nm}$ amorphous silicon $(\alpha-\mathrm{Si})$ layer was then deposited using low pressure chemical vapor deposition (LPCVD) at $560^{\circ} \mathrm{C}$. After deposition, some wafers were then implanted with fluorine at a dose of $2.5 \times 10^{15} \mathrm{~cm}^{-2}$ and an energy of $35 \mathrm{keV}$. After a short HF dip to remove any native oxide on the $\alpha$-Si surface, a $20 \mathrm{~nm} \mathrm{Ni}$ layer was deposited on a $\mathrm{F}$ implanted wafer and an unimplanted wafer and then patterned (dimensions $8 \mathrm{~mm} \times 10 \mu \mathrm{m}$ ) by lift-off (Fig. 1a) in preparation for MILC. On two other F implanted and unimplanted wafers, a $250 \mathrm{~nm}$ low temperature oxide (LTO) was deposited by LPCVD at $400^{\circ}$ C. Subsequently, the deposited oxide cap layer was patterned by photolithography and then wet etched to form a window $(360 \mu \mathrm{m} \times 360 \mu \mathrm{m})$ in the cap layer. After a short buffered 20:1 HF dip to remove any oxide, a $20 \mathrm{~nm}$ Ni layer was deposited on the wafers (Fig. 1b) but not patterned. The wafers were then diced into pieces and annealed in nitrogen for durations from 3 hours to 20 hours at a temperature in the range $550^{\circ} \mathrm{C}$ to $428^{\circ} \mathrm{C}$.

The samples were then characterized using Nomarski microscopy and Raman spectroscopy with a laser spot size of about $1 \mu \mathrm{m}$. For samples with an oxide cap, the $\mathrm{Ni}$ and oxide cap were removed by buffered HF before Raman measurements. The measured Raman intensity was normalized to the Raman intensity at a Raman shift of $520 \mathrm{~cm}^{-1}$.

\section{Results}

Fig. 2 shows optical Nomarski micrographs of unimplanted samples with no oxide cap (Fig. 2a) and with an oxide cap (Fig. 2b) after metal-induced lateral crystallization at $550^{\circ} \mathrm{C}$ for 15 hours. Lateral crystallization can be identified from the color contrast in the Si region adjacent to the Ni bar for the sample with no oxide cap, whilst a less clear color contrast is seen for the sample with an oxide cap. It can be clearly seen (color on-line) that the lateral crystallization length in the sample with no oxide cap is dramatically larger than that in the sample with an oxide cap. Measurements of the lateral crystallization length were made on 20 different locations and gave values of $74.6 \pm 0.5 \mu \mathrm{m}$ and $56.1 \pm 1.3 \mu \mathrm{m}$ for samples without and with an oxide cap respectively. These results show an increase of $33 \%$ in lateral crystallization length for the sample with no oxide cap.

Fig. 3 shows the lateral crystallization length as a function of anneal time for unimplanted samples with and without an oxide cap after metal-induced lateral crystallization at $550^{\circ} \mathrm{C}$. Measurements of lateral crystallization length were made on 20 different locations using an optical Nomarski microscope. It can be seen that longer lateral crystallization lengths are obtained for samples without an oxide cap at all anneal times. It should be mentioned that extrapolated values of crystallization length do not pass through the origin, which is very likely due to the influence of the ramp up time in the furnace.

Fig. 4 shows a graph of lateral crystallization length as a function of anneal temperature for unimplanted samples with and without an oxide cap after a 20 hour anneal at a temperature in the range $525^{\circ} \mathrm{C}$ to $428^{\circ} \mathrm{C}$. As expected, it can be seen that the lateral crystallization length decreases with anneal temperature for both types of sample. The samples without an oxide cap are seen to give larger values of

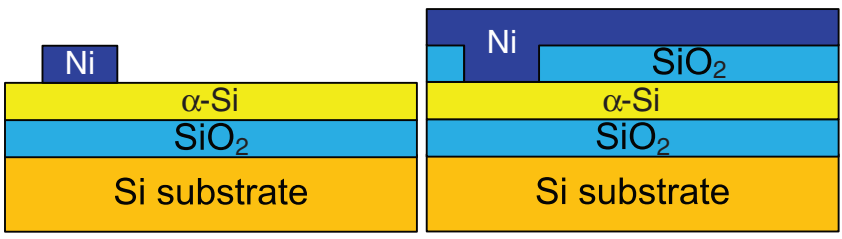

(a)

(b)

Figure 1. (Color online) Schematics of samples (a) without an oxide cap layer and (b) with an oxide cap layer. 

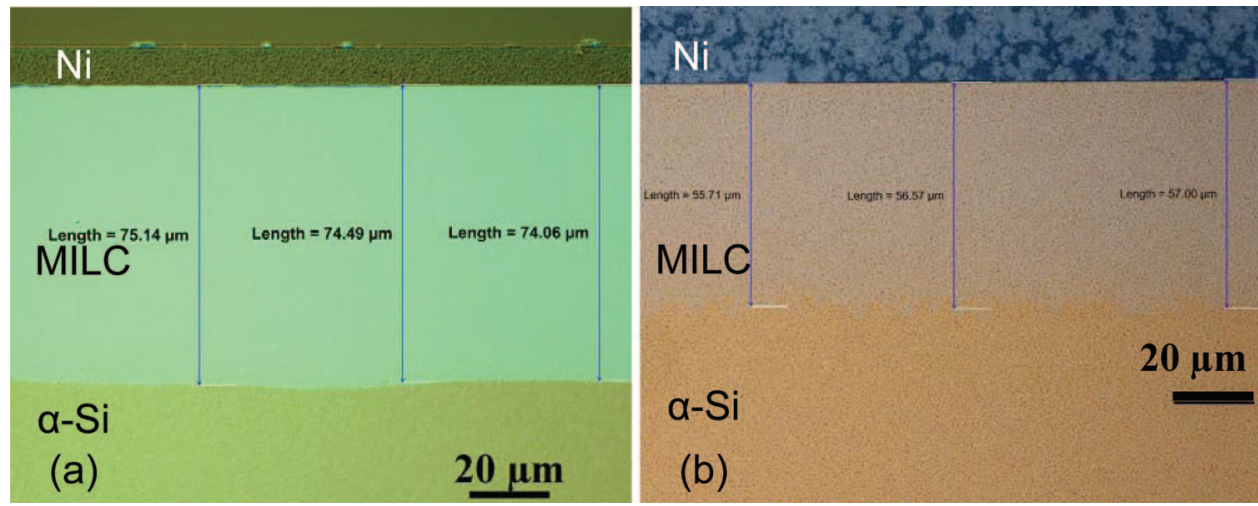

Figure 2. (Color online) Nomarski micrographs of metal-induced lateral crystallization (MILC) in $\alpha$-Si samples annealed at $550^{\circ} \mathrm{C}$ for 15 hours (a) without an oxide and (b) with an oxide cap layer.

lateral crystallization at anneal temperatures of $475^{\circ} \mathrm{C}, 500^{\circ} \mathrm{C}$ and $525^{\circ} \mathrm{C}$. At temperatures of $450^{\circ} \mathrm{C}$ and $400^{\circ} \mathrm{C}$, the oxide cap layer has no significant effect on the lateral crystallization length.

To further investigate the effect of the oxide cap layer, fluorine implanted samples are also studied as fluorine implant has been found to suppress random crystallization in amorphous silicon. ${ }^{2,16}$ Fig. 5 shows optical Nomarski micrographs of fluorine implanted samples without (Fig. 5a) and with an oxide cap (Fig. 5b) after metal-induced lateral crystallization at $550^{\circ} \mathrm{C}$ for 15 hours. Lateral crystallization can again be identified from the color contrast between the crystallized and amorphous regions. Measurements of crystallization length were made at 20 different locations and values of $84.1 \pm 0.3 \mu \mathrm{m}$ and 81.1 $\pm 1.3 \mu \mathrm{m}$ were obtained for samples without and with an oxide cap in fluorine implanted samples, respectively. Thus, crystallization length is only $3.7 \%$ larger in samples without an oxide cap.

To further investigate the effect of fluorine, Fig. 6 shows the lateral crystallization length as a function of anneal time for samples with and without an oxide cap after metal-induced lateral crystallization at $550^{\circ} \mathrm{C}$. Measurements of lateral crystallization length were made on 20 different locations using an optical Nomarski microscope. For a 15 hour anneal, the fluorine implant gives a dramatic $44.6 \%$ increase in crystallization length in samples with an oxide cap, from 56.1 \pm 1.3 to $81.1 \pm 1.3 \mu \mathrm{m}$. A similar, though smaller $(12.7 \%)$, increase in crystallization length, from $74.6 \pm 0.5$ to $84.1 \pm 0.3 \mu \mathrm{m}$, is also seen in samples without an oxide cap. Comparing the two fluorine implanted samples, the crystallization length in the sample without

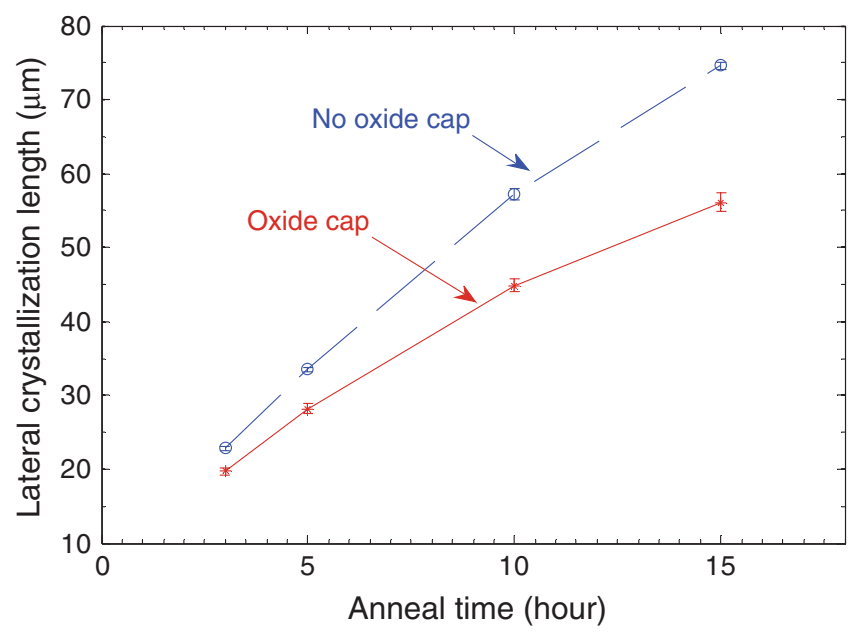

Figure 3. (Color online) Lateral crystallization length as a function of anneal time for $\alpha$-Si samples annealed at $550^{\circ} \mathrm{C}$ with and without an oxide cap layer an oxide cap is approximately $3.7 \%$ longer in the sample without an oxide cap. Thus the fluorine implant has some benefit on increasing the crystallization length even in samples without an oxide cap layer. For a 5 hour anneal, it is interesting to note that the crystallization length in the samples without an oxide cap is $8.9 \%$ shorter in the fluorine implanted samples.

To investigate the random crystallization regions of the film where MILC is not occurring, Raman spectroscopy was performed at locations far from the MILC region and normalized Raman spectra are presented in Fig. 7. For the unannealed sample, a Raman peak at $480 \mathrm{~cm}^{-1}$ can be clearly identified, indicating that the film is largely amorphous. ${ }^{10}$ After an anneal at $550^{\circ} \mathrm{C}$, the two $\mathrm{F}$ implanted samples again show strong Raman peaks at $480 \mathrm{~cm}^{-1}$, indicating that the films are still largely amorphous after anneal. In contrast for the samples without a fluorine implant, the Raman peaks at $480 \mathrm{~cm}^{-1}$ are dramatically smaller after anneal, indicating that there is less amorphous silicon present and hence that the anneal has given rise to considerable random crystallization. Comparing the unimplanted samples with and without an oxide cap, no Raman peak at $480 \mathrm{~cm}^{-1}$ can be identified for the sample with an oxide cap, whereas a small peak can be identified for the sample without an oxide cap. This result indicates that there is less amorphous silicon in the sample with an oxide cap and hence implies that the presence of an oxide cap increases the amount of random crystallization.

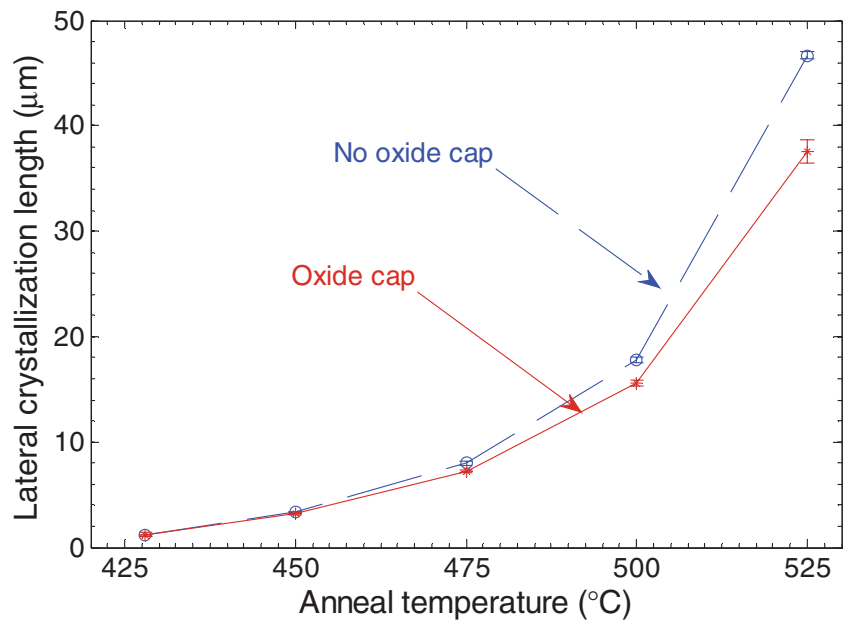

Figure 4. (Color online) Lateral crystallization length as a function of anneal temperature for $\alpha$-Si samples annealed for 20 hours with and without an oxide cap layer. 

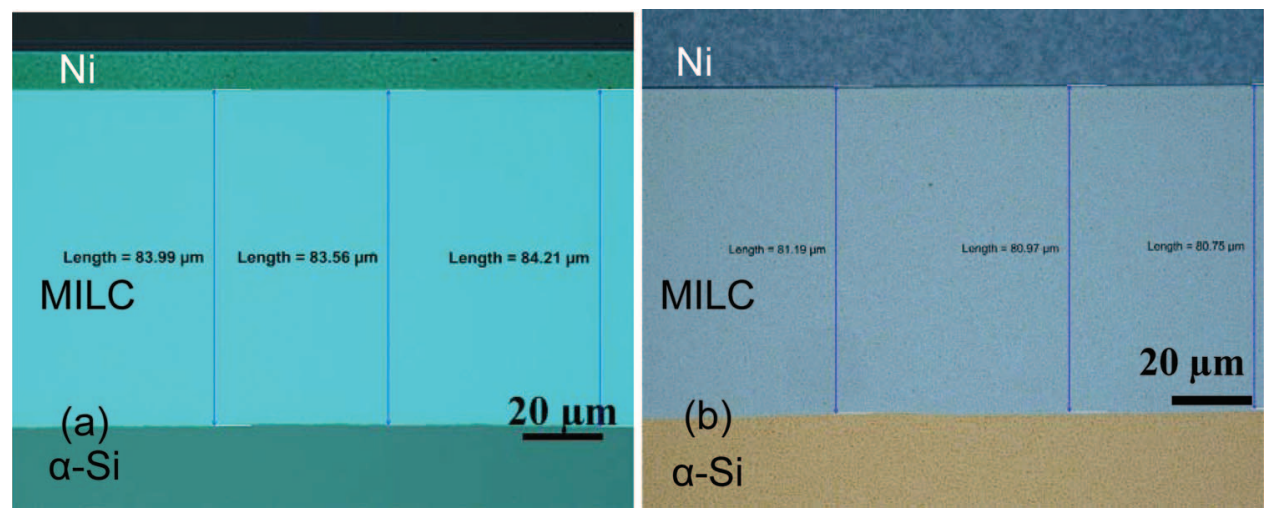

Figure 5. (Color online) Nomarski micrographs of fluorine implanted $\alpha$-Si samples annealed at $550^{\circ} \mathrm{C}$ for 15 hours (a) without an oxide cap layer and (b) with an oxide cap layer.

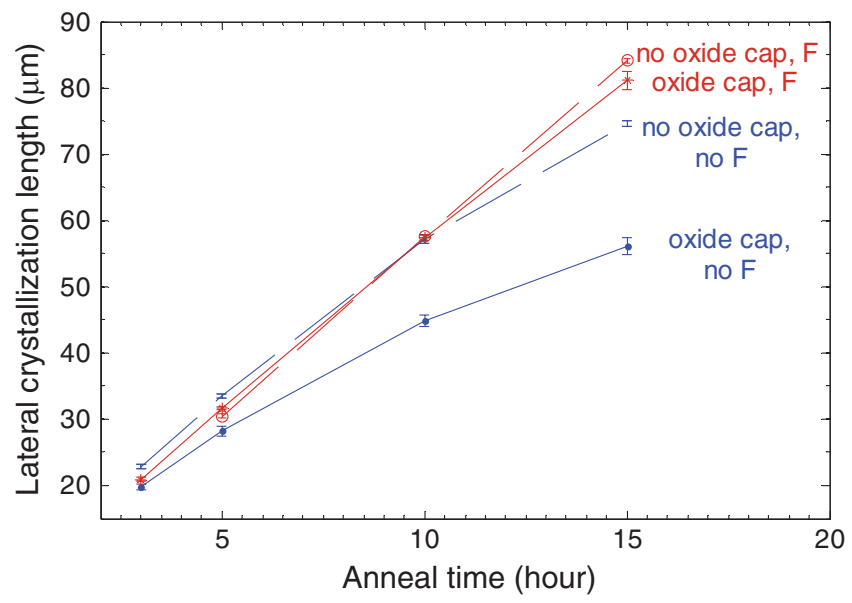

Figure 6. (Color online) Lateral crystallization length as a function of anneal time for $\alpha$-Si samples annealed at $550^{\circ} \mathrm{C}$ with and without an oxide cap layer and with and without a fluorine implant. Fluorine was implanted into $\alpha$-Si at a dose of $2.5 \times 10^{15} \mathrm{~cm}^{-2}$ and an energy of $35 \mathrm{keV}$.

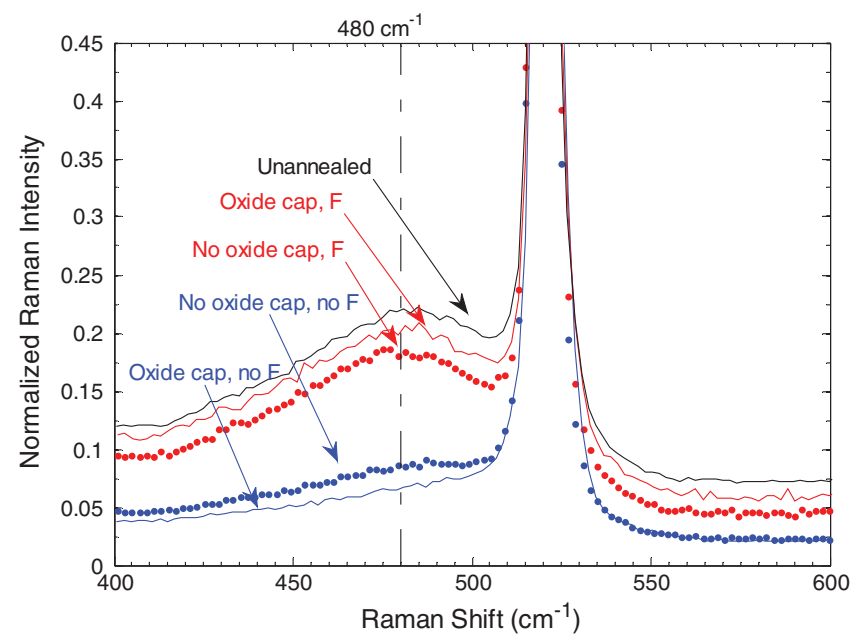

Figure 7. (Color online) Raman spectra measured in regions of $\alpha$-Si samples not covered by Ni and far away from the MILC regions. Results are show for samples far from the MILC region for $\alpha$-Si samples with and without an oxide cap layer and with and without a fluorine implant. The samples were annealed at $550^{\circ} \mathrm{C}$ for 15 hours. A Raman spectrum of a sample before anneal is also given for comparison.

\section{Discussion}

We first discuss the results in Fig. 3, which show the effect of a capping oxide on unimplanted samples. Cheng et $\mathrm{al}^{17}$ reported that lateral crystallization is slowed and even stopped by random crystallization and Ryu et $\mathrm{l}^{18}$ reported that random crystallization starts preferentially at the interface between amorphous silicon and the underlying $\mathrm{SiO}_{2}$. This mechanism could explain why samples without an oxide cap give longer lateral crystallization lengths than samples with an oxide cap. For samples without an oxide cap (Fig. 8a), random grains only grow from the interface between the $\alpha$-Si and the underlying oxide, whereas for samples with an oxide cap (Fig. 8b), random grains grow from both the bottom and top $\alpha$-Si interfaces. Therefore, more random grain nucleation is expected in $\alpha$-Si samples with an oxide cap than without an oxide cap, which therefore explains the longer lateral crystallization lengths seen in samples without an oxide cap. At lower anneal temperatures (below $475^{\circ} \mathrm{C}$ ), random crystallization is significantly reduced ${ }^{19}$ and hence the detrimental effect of the oxide cap is greatly reduced, as seen in Fig. 4.

Support for the above explanation also comes from the results on fluorine-implanted samples shown in Fig. 6. Our earlier research ${ }^{2,16}$ showed that fluorine enhances the crystallization length in amorphous silicon sheets and nanowires due to the suppression of random grain nucleation at the interface between the amorphous silicon and the underlying silicon dioxide layer. This mechanism also explains the results in Fig. 6, where a large increase in crystallization length is obtained when fluorine is implanted into samples with an oxide cap layer. The $44.6 \%$ increase in crystallization length after a 15 hour anneal can be explained by the action of the fluorine in suppressing random grain nucleation at both the top and bottom silicon dioxide interfaces. The smaller $12.7 \%$ increase in crystallization length seen in the samples without an oxide cap can be explained by noting that the fluorine only suppresses random grain nucleation at the bottom silicon dioxide interface. There is no silicon dioxide layer at the top

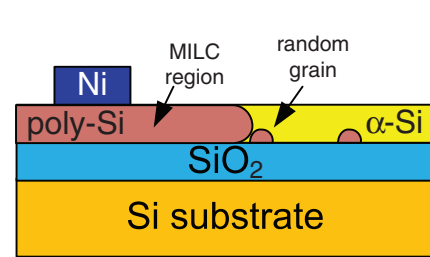

(a)

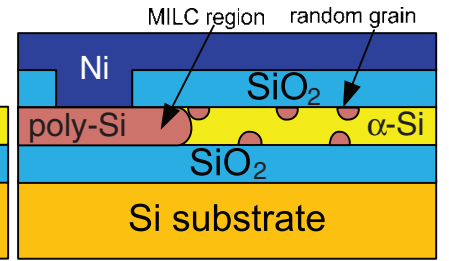

(b)
Figure 8. (Color online) Schematics showing metal-induced lateral crystallization and random grain nucleation in samples (a) without an oxide cap layer and (b) with an oxide cap layer. 
interface in these samples and hence there is no benefit from the fluorine implant at this interface.

If fluorine totally suppressed random grain nucleation at the silicon dioxide interfaces, it might be expected that the crystallization lengths in fluorine implanted samples with and without a cap layer would be the same. However, this is not the case, as can be seen in Fig. 6 for a 15 hour anneal at $550^{\circ} \mathrm{C}$. The crystallization length in the fluorine implanted sample without a cap layer is approximately $3.7 \%$ longer than in the sample with the oxide cap. The fluorine implant was performed before the deposition of the oxide cap and the implantation energy was chosen to give a fluorine peak at the bottom amorphous silicon interface. It is likely therefore that there is insufficient fluorine at the top $\alpha$-Si interface to completely suppress random grain nucleation from the top interface in samples with an oxide cap. A lower fluorine implantation energy would increase the fluorine concentration at the top $\alpha-\mathrm{Si}$ interface and may give improved suppression of grain nucleation at this interface. Alternatively, it is possible that the $400^{\circ} \mathrm{C}$ oxide deposition could have induced some additional random grain nucleation in the samples with an oxide cap. However our earlier work $^{2}$ indicated that random grain nucleation was not significant at temperatures below $500^{\circ} \mathrm{C}$. Furthermore, fluorine diffusion in amorphous silicon is not significant at temperatures below $500{ }^{\circ} \mathrm{C},{ }^{20}$ so it is unlikely that the $400^{\circ} \mathrm{C}$ oxide deposition is responsible for the $3.7 \%$ longer crystallization length in fluorine implanted samples with an oxide cap.

At short anneal times, the results in Fig. 6 show that the crystallization length in the samples without an oxide cap is shorter for the fluorine implanted samples. This is an interesting result and suggests that a second mechanism is influencing the crystallization behavior in fluorine implanted samples. It is possible that fluorine may slow down silicon diffusion and thereby reduce the crystallization length. Further work is needed to investigate this effect.

\section{Conclusions}

In this work, we have investigated the effect of an oxide cap on the metal-induced lateral crystallization of amorphous silicon. Amorphous silicon without an oxide cap gave a 33\% increase in lateral crystallization length after an anneal of 15 hours at $550^{\circ} \mathrm{C}$ compared with amorphous silicon with an oxide cap. $\mathrm{A} \approx 10 \%$ increase was obtained at temperatures of $500^{\circ} \mathrm{C}$ and $475^{\circ} \mathrm{C}$, but no increase at lower temperatures. The increase in lateral crystallization length for samples without oxide cap has been explained by the elimination of random grain crystallization at the interface between $\alpha-\mathrm{Si}$ and the oxide cap. It has also been demonstrated that fluorine implantation into the amorphous silicon can be used to suppress random grain nucleation at the interface between the $\alpha$-Si and the oxide cap, giving lateral crystallization lengths almost equivalent to those in samples without an oxide cap.

\section{References}

1. C. W. Chang, S. F. Chen, C. L. Chang, C. K. Deng, J. J. Huang, and T. F. Lei, Ieee Electr. Device L, 29(5), 474 (2008).

2. K. Sun, M. M. A. Hakim, R. Gunn, and P. Ashburn, ECS J. Solid State Sci. Technol., 1(2), P94 (2012)

3. K. Sun, M. M. A. Hakim, and P. Ashburn, Electrochem. Solid St., 15(3), H62 (2012).

4. C. Hayzelden, J. L. Batstone, and R. C. Cammarata, Appl. Phys. Lett., 60(2), 225 (1992).

5. C. C. Liao, M. C. Lin, S. X. Liu, and T. S. Chao, Ieee Electr. Device L, 33(2), 239 (2012).

6. C. P. Chang and Y. S. Wu, J. Electrochem. Soc., 157(2), H192 (2010)

7. G. B. Kim, Y. G. Yoon, M. S. Kim, H. Jung, S. W. Lee, and S. K. Joo, Ieee T Electron. Dev., 50(12), 2344 (2003).

8. M. Wong, Z. H. Jin, G. A. Bhat, P. C. Wong, and H. S. Kwok, Ieee T Electron. Dev., 47(5), 1061 (2000).

9. C. P. Chang and Y. C. S. Wu, Ieee Electr. Device. L, 28(11), 990 (2007)

10. C. P. Chang and Y. S. Wu, Ieee Electr. Device. L, 30(11), 1176 (2009).

11. Y. C. Wu, T. C. Chang, C. W. Chou, Y. C. Wu, P. T. Liu, C. H. Tu, W. J. Huang, J. C. Lou, and C. Y. Chang, J. Electrochem. Soc., 152(7), G545 (2005).

12. W. M. Cheung, C. F. Cheng, M. C. Poon, C. W. Kok, and M. Chan, 2002 Ieee Hong Kong Electron Devices Meeting, Proceedings, 27-30 (2002).

13. C. Tao, R. Ishihara, W. Metselaar, K. Beenakker, and M. Y. Wu, Jpn. J. Appl. Phys., 47(3), 1880 (2008).

14. J. H. Ahn, J. H. Eom, and B. T. Ahn, J. Electrochem. Soc., 151(6), H141 (2004)

15. T. F. Ma and M. Wong, J. Appl. Phys., 91(3), 1236 (2002).

16. M. M. A. Hakim and P. Ashburn, J. Electrochem. Soc., 154(8), H734 (2007).

17. C. F. Cheng, T. C. Leung, M. C. Poon, C. W. Kok, and M. S. Chan, Ieee T Electron. Dev., 51(12), 2205 (2004).

18. M. K. Ryu, S. M. Hwang, T. H. Kim, K. B. Kim, and S. H. Min, Appl. Phys. Lett., 71(21), 3063 (1997)

19. C. Spinella, S. Lombardo, and F. Priolo, J. Appl. Phys., 84(10), 5383 (1998).

20. S. P. Jeng, T. P. Ma, R. Canteri, M. Anderle, and G. W. Rubloff, Appl. Phys. Lett., 61(11), 1310 (1992) 